\title{
Bursty emission of whistler waves in association with plasmoid collision
}

\author{
Keizo Fujimoto ${ }^{1,2}$ \\ ${ }^{1}$ School of Space and Environment, Beihang University, Beijing, China \\ ${ }^{2}$ Division of Theoretical Astronomy, National Astronomical Observatory of Japan, Mitaka, Tokyo, Japan \\ Correspondence to: Keizo Fujimoto (fujimoto@buaa.edu.cn)
}

Received: 21 April 2017 - Revised: 29 May 2017 - Accepted: 21 June 2017 - Published: 31 July 2017

\begin{abstract}
A new mechanism to generate whistler waves in the course of collisionless magnetic reconnection is proposed. It is found that intense whistler emissions occur in association with plasmoid collisions. The key processes are strong perpendicular heating of the electrons through a secondary magnetic reconnection during plasmoid collision and the subsequent compression of the ambient magnetic field, leading to whistler instability due to the electron temperature anisotropy. The emissions have a bursty nature, completing in a short time within the ion timescales, as has often been observed in the Earth's magnetosphere. The whistler waves can accelerate the electrons in the parallel direction, contributing to the generation of high-energy electrons. The present study suggests that the bursty emission of whistler waves could be an indicator of plasmoid collisions and the associated particle energization during collisionless magnetic reconnection.
\end{abstract}

Keywords. Magnetospheric physics (plasma waves and instabilities) and space plasma physics (magnetic reconnection; numerical simulation studies)

\section{Introduction}

Whistler waves are fundamental plasma waves frequently observed in space in association with transient phenomena, such as collisionless shocks (e.g., Olson et al., 1969; Rodriguez and Gurnett, 1975; Lengyel-Frey et al., 1996; Zhang et al., 1999; Hull et al., 2012) and magnetic reconnection (e.g., Deng and Matsumoto, 2001; Wei et al., 2007; Tang et al., 2013; Graham et al., 2016; Huang et al., 2016; Zhao et al., 2016; Uchino et al., 2017). The waves have a righthand polarization with respect to the ambient magnetic field, so they can couple with the electrons through the cyclotron resonance and give rise to pitch-angle scattering and parallel acceleration (e.g., Kennel and Petschek, 1966; Gary and Wang, 1996; Schreiner et al., 2017). Such microscopic waveparticle interactions can cause anomalous transport in momentum and energy, resulting in anomalous magnetic dissipation in collisionless plasma where the classical Coulomb collision is negligibly weak. Regardless of the potential importance of whistler waves in collisionless plasma, the generation mechanism in transient configurations has been poorly understood.

Satellite observations have shown that whistler emissions usually have bursty properties continuing for a very short time compared to the dynamical timescales that are usually much longer than the ion timescales, such as the Alfvén transit time across the boundary layers. There are two explanations for these observations: the first is that the emissions are very localized in space, and the second is that they are intermittent within a short timescale. So far, a number of kinetic simulations have been carried out to investigate how whistler waves can be generated and what their roles are in the dynamical processes that control macroscopic configurations (e.g., Hellinger et al., 1996; Scholer et al., 2003; Fujimoto and Sydora, 2008; Fujimoto, 2014; Goldman et al., 2014; Burgess and Scholer, 2015). In particular, collisionless magnetic reconnection enables an explosive release of magnetic field energy into plasma kinetic energy. The associated plasma jets and pressure anisotropy can boost the activities of a variety of plasma waves, including whistler waves, which can in turn have an impact on the reconnection process through magnetic dissipation and plasma heating (Fujimoto et al., 2011). 
Previous kinetic simulations of collisionless reconnection have suggested that whistler waves are generated in the magnetic field pileup region downstream of the $\mathrm{x}$-line (Fujimoto and Sydora, 2008) and in the separatrix region separating the inflow and outflow regions (Fujimoto, 2014; Goldman et al., 2014). In the pileup region, the electrons are heated preferentially in the perpendicular direction due to betatron acceleration and have a temperature anisotropy leading to the whistler emission. Meanwhile, in the separatrix region, the electrons are strongly accelerated in the parallel direction due to a double layer formed locally in the separatrix region. The intense electron beam can be coupled with the stationary background electrons, which directly (Fujimoto, 2014) or indirectly (Goldman et al., 2014) triggers obliquely propagating whistlers. In both regions, the whistler emissions occur within a very narrow extent across the field lines, which explains the bursty nature in the observations. However, these models were based on a steady-state x-line configuration formed around the dissipation region relatively at the beginning of the reconnection process.

Recent large-scale and long-term particle-in-cell (PIC) simulations have shown the dynamical evolution of the reconnection current layer, repeating the elongation in the downstream direction (Fujimoto, 2006) and the formation of plasmoids (Daughton and Karimabadi, 2007). In fact, a chain of plasmoids and flux ropes has often been observed in association with reconnection and accompanied by energetic particles and intense wave activities, including whistlers (Chen et al., 2008; Wang et al., 2016; Zhao et al., 2016). In this paper, a new generation mechanism for whistler waves is proposed in association with plasmoid collision through the use of large-scale PIC simulation. Contrary to previous scenarios in the magnetic field pileup region and separatrix region, the present model provides a short-term emission only after the plasmoid collision has been completed. The resultant whistler emission is bursty and has characteristics different from other emissions, so it can work as an indicator of plasmoid and flux rope collisions.

\section{Simulation model}

The simulations are carried out through the use of a twodimensional (2-D) electromagnetic PIC model with the adaptive mesh refinement (AMR) and particle splittingcoalescence method (Fujimoto, 2011). The refinement criteria for the AMR are provided by the local electron Debye length $\lambda_{\text {De }}$ and the out-of-plane electron flow velocity $V_{\mathrm{e} y}$ defined at the center of each computational cell. Each cell is subdivided when $\Delta_{\mathrm{L}} \geq 2.0 \lambda_{\mathrm{De}}$ or $V_{\mathrm{e} y} \geq 2.0 V_{\mathrm{A}}$ is satisfied; otherwise the child cells are removed when the computational cells are squares with a size of $\Delta_{\mathrm{L}}$, and $V_{\mathrm{A}}$ is the Alfvén velocity based on the asymptotic upstream magnetic field $B_{0}$ and the plasma density $n_{0}$ at the center of the initial current sheet. The system boundaries are "open" in both the inflow and outflow directions (Fujimoto, 2014).

The initial current sheet profile employs a Harris-type equilibrium with the magnetic field $B_{x}(z)=-B_{0} \tanh (z / \delta)$ and the number density $n(z)=n_{0} \operatorname{sech}^{2}(z / \delta)+n_{\mathrm{b}} \tanh ^{2}(z / \delta)$, where $\delta$ is the half width of the current sheet and the subscript $\mathrm{b}$ indicates the background parameter. We choose $\delta=\lambda_{\mathrm{i}}$ and $n_{\mathrm{b}}=0.044 n_{0}$ with $\lambda_{\mathrm{i}}$ as the ion inertia length based on $n_{0}$. Note that the background plasma is removed in the center of the current sheet in order to avoid artificial streaming instabilities (e.g., a two-stream instability between the plasma sheet and background components). Although such a density profile causes a weak pressure imbalance, the equilibrium is quickly established without any significant modification to the current sheet structure. The ion-to-electron mass ratio and velocity of light are $m_{\mathrm{i}} / m_{\mathrm{e}}=100$ and $c / V_{\mathrm{A}}=33$, respectively, corresponding to $\omega_{\mathrm{pe}} / \omega_{\mathrm{ce}}=3.3$, where $\omega_{\mathrm{pe}}$ and $\omega_{\text {ce }}$ are the electron plasma frequency and the cyclotron frequency based on $n_{0}$ and $B_{0}$, respectively. The temperature ratios are $T_{0 \mathrm{i}} / T_{0 \mathrm{e}}=3.0, T_{\mathrm{bi}} / T_{\mathrm{be}}=1.0$, and $T_{\mathrm{be}} / T_{0 \mathrm{e}}=1.0 \mathrm{so}$ that the background ions are colder than the plasma sheet ions. The system size is $L_{x} \times L_{z}=655 \lambda_{\mathrm{i}} \times 328 \lambda_{\mathrm{i}}$, which is entirely covered by base-level cells (coarsest cells) with $\Delta_{L_{\mathrm{b}}}=0.08 \lambda_{\mathrm{i}}$ and can be subdivided locally into finer cells up to the dynamic range level with $\Delta_{L_{\mathrm{D}}}=0.02 \lambda_{\mathrm{i}}$. Therefore, the highest spatial resolution is $32768 \times 16384$ (the effective number of the finest cells when they cover the whole domain) and the maximum number of particles used is $\sim 10^{10}$ for each species. The normalization parameters in the current study are $m_{\mathrm{i}}$ for mass, $e$ for charge, $\lambda_{\mathrm{i}}$ for length, and $V_{\mathrm{A}}$ for velocity, unless otherwise noted.

\section{Results}

The present study uses a small magnetic perturbation in order to initiate magnetic reconnection so that the $\mathrm{x}$-line is generated at the center of the system (Fujimoto, 2006). Once the simulation has started, a fast reconnection is soon achieved with a rate of $E_{\mathrm{R}} \approx 0.12$, where $E_{\mathrm{R}}$ is evaluated by $E_{y}$ at the most dominant $\mathrm{x}$-line normalized to the upstream values. In the quasi-steady phase, the electron current layer formed around the $\mathrm{x}$-line is elongated in the outflow direction and is subject to plasmoid formations. By repeating the electron layer elongation and plasmoid ejection, the reconnection exhaust expands to a distance far downstream of the $x$-line. Figure $1 \mathrm{a}-\mathrm{c}$ show the evolution of a plasmoid indicated by the black arrow in the exhaust. This plasmoid has been ejected in the $-x$ direction and eventually merged with the initial current sheet at the downstream edge of the exhaust. In association with the collision with the downstream current sheet, a secondary reconnection takes place between the field lines directing downward $(-z)$ in the plasmoid and upward $(+z)$ in the downstream current sheet. During the secondary reconnection, the electrons are strongly accelerated in the vicinity 
of the $x$-line in the out-of-plane $(-y)$ direction (see Fig. 1a and $b$ ). Note that this direction is opposite to that in the main reconnection layer.

The localized structure of the secondary reconnection region is shown in Fig. 1d-f. The reconnection outflow jets associated with the plasmoid collision are ejected mainly in the $z$ direction (Fig. 1d). Because the plasmoid scale is comparable to the local ion gyroradius, the electrons are more effectively accelerated than the ions. The ejected electrons are partially magnetized in the upper $(+z)$ and lower $(-z)$ regions of the secondary $x$-line, so the electrons are selectively heated in the perpendicular direction, resulting in $T_{\mathrm{e} \perp} / T_{\mathrm{e} \|}>$ 1 (Fig. 1e). The perpendicular heating is also significant in the pileup region further downstream of the exhaust, as described in Fujimoto and Sydora (2008). In Fig. 1g-i, the localized structure is presented after the secondary reconnection has ended. Since reconnection is over, the outflow jets do not exist (Fig. 1g). However, the intense temperature anisotropy of the electrons with $T_{\mathrm{e} \perp} / T_{\mathrm{e} \|}>1$ still remains off the Equator around the downstream edge of the exhaust (Fig. 1h). This is because the perpendicularly heated electrons, due to the reconnection process, move further downstream almost together with the field lines. At this time, an intense emission of microscopic waves with the wavelength $\lambda \approx 7 \lambda_{\mathrm{e}}^{*}$ occurs off the Equator plane, where $\lambda_{\mathrm{e}}^{*}$ is the local electron inertia length. The waves typically appear in $E_{y}$, the out-of-plane electric field (Fig. 1i), which indicates that they are dominated by the electromagnetic properties. The emission is also evident in Movie S1 in the Supplement. It is interesting to note that the waves are not clearly excited during the secondary reconnection (Fig. 1f), regardless of the strong electron acceleration in the vicinity of the $x$-line.

Figure 2 shows the wave properties arising in $E_{y}$. One can see from Fig. 2a that the microscopic waves are excited at $l / \lambda_{\mathrm{i}} \approx \pm 10$ and propagate along the field line away from the Equator point of $l=0$. Here, $l$ represents the coordinate along a field line traced from $(x, z)=(0,-18.5)$, which passes through the wave active region. Since the starting point of the field line is far away from the downstream edge of the exhaust $(x \approx 150$ at $t=114)$, the field line at each time step is considered to be almost identical. The examples of the field line at $t \omega_{\mathrm{ci}}=106$ and 114 are indicated with dashed curves in Fig. If and i, respectively. It is found that the wave emission is bursty: the emission of microscopic waves with $k_{\|} \lambda_{\mathrm{e}}^{*}>0.5$ is only significant around $112<t \omega_{\mathrm{ci}}<116$ (Fig. 2b), which is a much shorter time compared to the dynamical scale, for instance, of the transit time of the plasmoid across the exhaust $\left(\sim 30 \omega_{\mathrm{ci}}^{-1}\right.$ for the exhaust with a size of $\sim 100 \lambda_{\mathrm{i}}$ in the $x$ direction).

To identify the generation mechanism of the waves, we compare the wave spectrum in the simulation with the dispersion relation derived from linear theory. The wave spectrum presented in Fig. 2c (color contour) shows a clear peak at $\omega \approx 0.5 \omega_{\mathrm{ce}}^{*}$ and $\left|k_{\|} \lambda_{\mathrm{e}}^{*}\right| \approx 0.9$ for the electromagnetic waves, which is consistent with the typical characteristics of whistler waves, where $\omega_{\mathrm{ce}}^{*}$ is the local electron cyclotron frequency. The electric field data for calculating the wave spectrum are taken from the base-level cells of the AMR hierarchy. It is known that the wave dispersion depends on the grid size of the numerical simulation (Birdsall and Langdon, 1991), i.e., on the cell level of the AMR. However, this dependency is only remarkable for the grid-scale waves with $\lambda \approx \Delta_{L_{\mathrm{b}}}$. The dominant waves in the current analysis have a scale of $\lambda \approx 7 \lambda_{\mathrm{e}}^{*} \approx 23 \Delta_{L_{\mathrm{b}}}$ based on the local density $n_{\mathrm{e}} \approx 0.15 n_{0}$. This indicates that the current waves of interest have a much larger scale than the base-level cell size, so the gridding effect on the wave dispersion is negligible.

The dispersion curve for the whistler waves excited by the temperature anisotropy is superposed on the simulation result in Fig. 2c. The wave dispersion is obtained from the linearized Vlasov equation for the electrons with temperature anisotropy (Gary, 1993) so that

$X^{2}-\frac{1}{\Omega^{2}} Y^{2}-\frac{Y}{X \sqrt{\beta_{\mathrm{e} \|}}} Z(\xi)+\frac{1}{2}\left(\frac{T_{\mathrm{e} \perp}}{T_{\mathrm{e} \|}}-1\right) Z^{\prime}(\xi)=0$,

where $X=k_{\|} \lambda_{\mathrm{e}}^{*}, \quad Y=\omega / \omega_{\mathrm{ce}}^{*}$ with $\omega=\omega_{r}+i \gamma, \quad \Omega=$ $\omega_{\mathrm{pe}}^{*} / \omega_{\mathrm{ce}}^{*}, \quad Z(\xi)=1 / \sqrt{\pi} \int_{-\infty}^{\infty} \exp \left(-x^{2}\right) /(x-\xi) \mathrm{d} x$ is the plasma dispersion function with $\xi=(Y-1) / X \sqrt{\beta_{\mathrm{e} \|}}$, and $\beta_{\mathrm{e} \|}$ is the plasma beta based on the electron parallel pressure. The parameters used for calculating the dispersion curve are $T_{\mathrm{e} \perp} / T_{\mathrm{e} \|}=2.5, \beta_{\mathrm{e} \|}=0.22$, and $\Omega=1.9$, which are taken at the wave active region in the simulation (i.e., inside the black dashed box in Fig. 2a). Figure 2c demonstrates that the simulation result is consistent with the linear dispersion, suggesting that the microscopic wave burst observed in the simulation is due to the whistler instability driven by the electron temperature anisotropy.

The temperature anisotropy is generated through the secondary reconnection in association with plasmoid collision. As shown in Fig. 3a, the key process leading to the temperature anisotropy is the intense perpendicular heating in the downstream region of the electron jets (indicated by sky blue arrows). This process is similar to that in the previous study (Fujimoto and Sydora, 2008), in which whistler waves are excited in the pileup region formed downstream of the main reconnection $\mathrm{x}$-line. However, in the current simulation, we find that the whistler emission is not clear at this time. This is partly because the pileup process of the field lines is weak in the region downstream of the secondary $\mathrm{x}$-line, so the magnetic field intensity remains low and insufficient for the highenergy electrons to be completely magnetized. Furthermore, the field line structure, including the out-of-plane Hall field (not shown), is highly nonuniform in this region in the scale below the ion inertia length. Therefore, the dispersion relation Eq. (1) derived for magnetized electrons under a uniform magnetic field is no longer valid in this region.

After the plasmoid merging and the associated reconnection process have been completed, the electrons with temperature anisotropy move further downstream of the main 

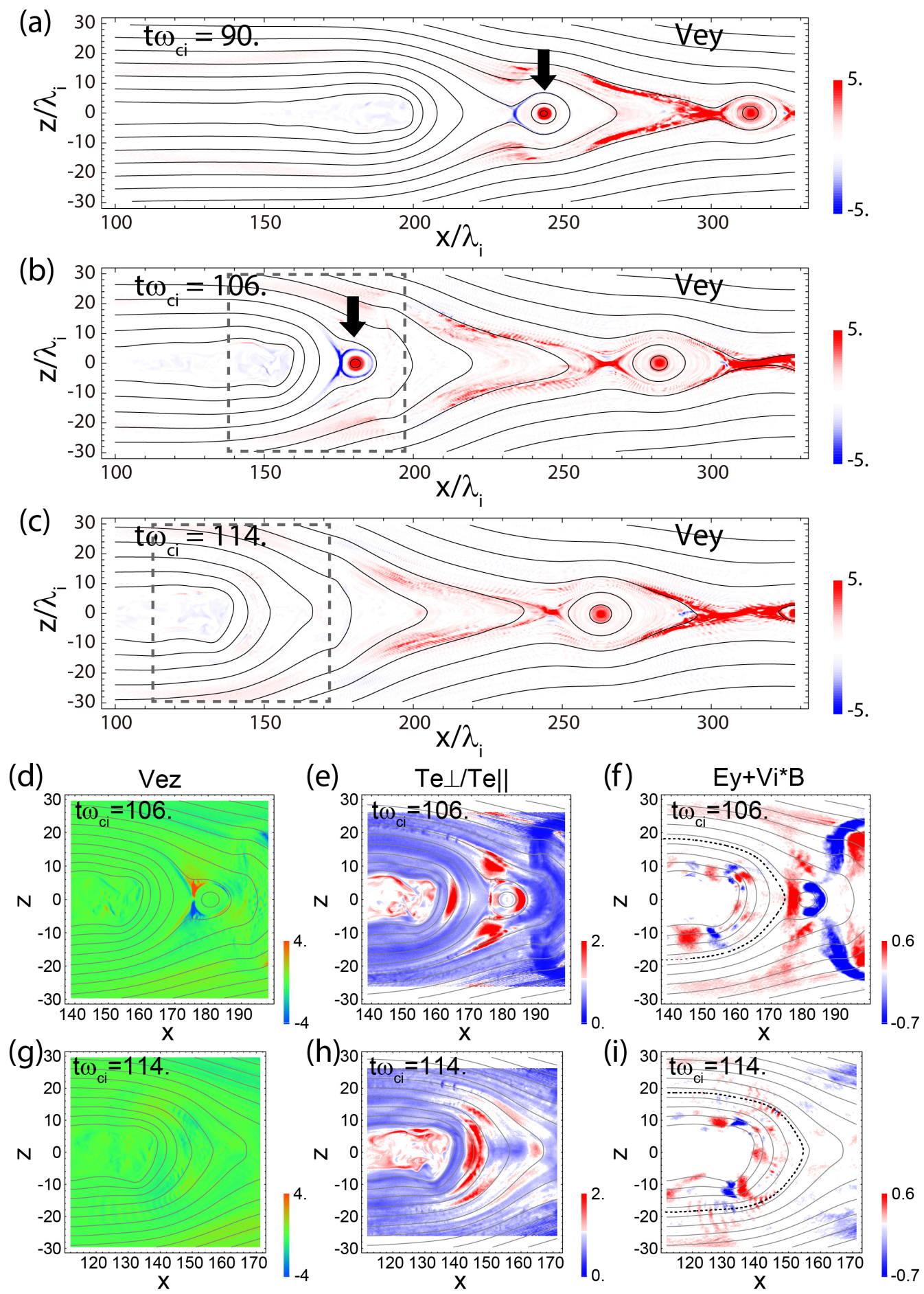

Figure 1. Time evolution of the main reconnection exhaust. (a-c) Two-dimensional snapshots of the out-of-plane electron bulk velocity at (a) $t \omega_{\mathrm{ci}}=90$, (b) 106 , and (c) 114 . (d-f) Localized profiles at $t \omega_{\mathrm{ci}}=106$ for (d) $V_{\mathrm{e} z}$, (e) $T_{\mathrm{e} \perp} / T_{\mathrm{e} \|}$, and (f) $E_{y}+\left(V_{\mathrm{i}} \times B\right)_{y}$. (g-i) The same profiles as in (d-f) but in a different area at $t \omega_{\mathrm{ci}}=114$. The black solid curves $(\mathbf{a}-\mathbf{c})$ and gray solid curves $(\mathbf{d}-\mathbf{i})$ represent the magnetic field lines, and the gray dashed boxes in (b) and (c) indicate the areas shown in (d-f) and (g-i), respectively. The black dashed curves in (f) and (i) are the field lines traced at each time step from $(x, z)=(0,-18.5)$, a point far away from the areas in (f) and (i). 

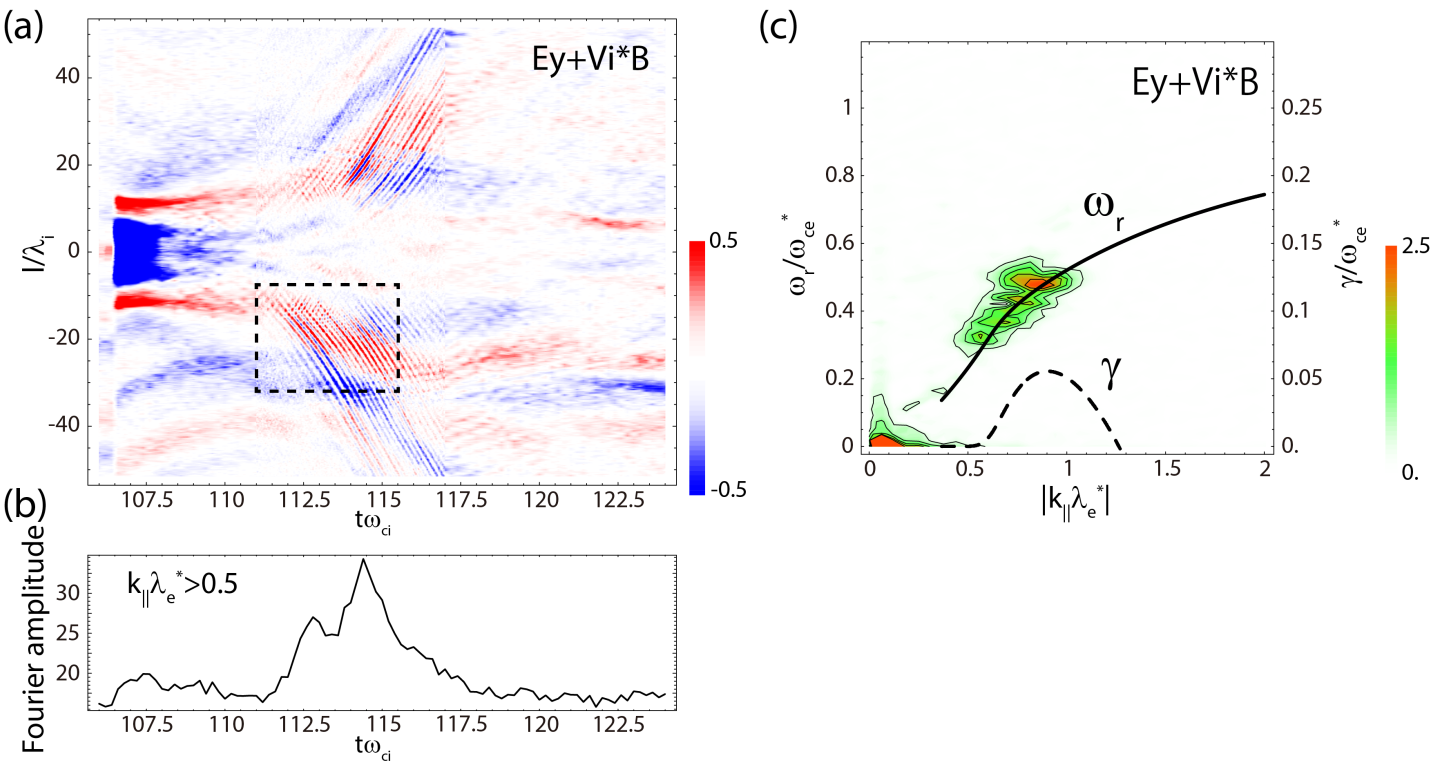

Figure 2. Properties of the electromagnetic waves propagating along the field lines. (a) Time evolution of $E_{y}+\left(V_{\mathrm{i}} \times B\right)_{y}$ along the field line traced from $(x, z)=(0,-18.5)$ at each time step. The vertical axis is the field-aligned coordinate with $l=0$ at the Equator point where $B_{x}=0$ is satisfied. (b) Fourier amplitude calculated along the field line and averaged over $k_{\|} \lambda_{\mathrm{e}}^{*}>0.5$ at each time step, where $\lambda_{\mathrm{e}}^{*}$ is the local electron inertia length. (c) The wave spectrum (color contour) of $E_{y}+\left(V_{\mathrm{i}} \times B\right)_{y}$ in the $\omega_{r}-k_{\|}$space for the area indicated by the black dashed box in (a). The theoretical curves of the dispersion relation Eq. (1) for the whistler instability driven by the electron temperature anisotropy are superposed by a solid curve for $\omega_{r}$, the real frequency, and by a dashed curve for $\gamma$, the growth rate.

(a)

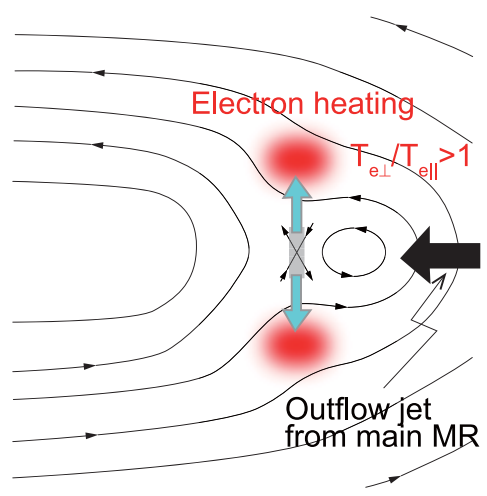

(b)

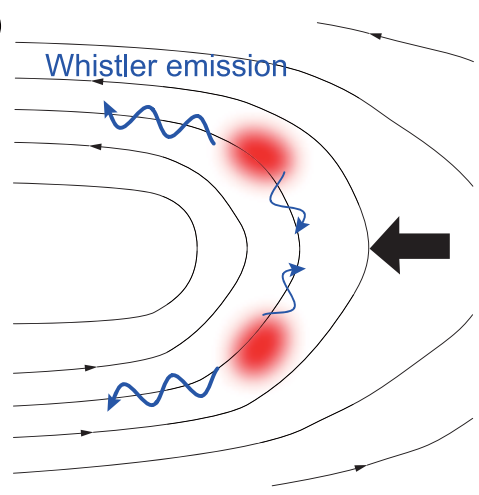

Figure 3. Schematic diagram showing the generation mechanism of a bursty emission of whistler waves in association with plasmoid collision. (a) When plasmoid collision occurs in the downstream edge of the main reconnection exhaust, a secondary reconnection takes place. The electron outflow jets (sky blue arrows) are generated in the $z$ direction, leading to the perpendicular heating (red filled circles) of the electrons in the regions downstream of the secondary $\mathrm{x}$-line. (b) As the plasmoid merging proceeds, the perpendicularly heated electrons move to the edge of the larger plasmoid (current sheet) and are compressed due to the magnetic tension force. As a result, the electrons become strongly magnetized so that a favorable condition for the whistler emission (blue arrows) is produced.

reconnection jet. The temperature anisotropy is almost preserved during convection because the whistler emission that can relax the anisotropy is absent. The electrons finally reach the edge of the downstream current sheet and are compressed at the off-Equator regions (Fig. 3b) due to the pileup of the magnetic field. The whistler waves are triggered at this moment and propagate along the field line. Once the whistler waves are excited, the temperature anisotropy is quickly de- creased due to the pitch-angle scattering, and the wave emissions are suppressed. The timescale of the isotropization is estimated as $t \omega_{\mathrm{pe}}^{*} \sim 40$ (Ossakow et al., 1972), which corresponds to $t \omega_{\mathrm{ci}} \sim 0.3$ for the local parameters $\left(n=0.15 n_{0}\right.$ and $\boldsymbol{B}=0.67 B_{0}$ ) in the current simulation. Therefore, the whistler emissions associated with the plasmoid collision are very bursty with a timescale smaller than the typical ion timescales. 
(a)

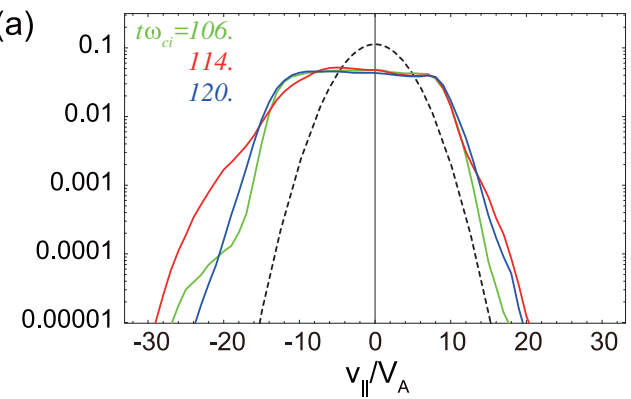

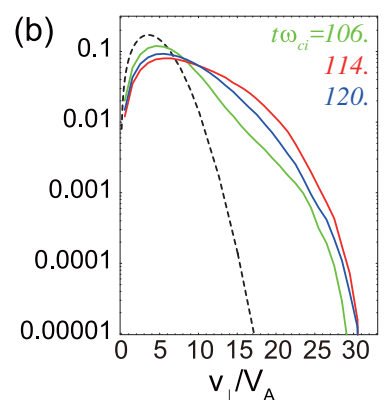

Figure 4. Reduced distribution functions of the electrons in (a) parallel and (b) perpendicular directions. The distribution functions are averaged over the field line $\left(-50 \leq l / \lambda_{\mathrm{i}} \leq 0\right)$ traced from $(x, z)=(0,-18.5)$ at $t \omega_{\mathrm{ci}}=106$ (green curves), 114 (red curves), and 120 (blue curves). The dashed curves indicate the distribution function of the initial background (lobe) electrons.

During the whistler emissions, the electrons can be accelerated in the parallel direction. Figure 4 shows the electron distribution functions averaged over the field line $(-50 \leq$ $\left.l / \lambda_{\mathrm{i}} \leq 0\right)$ traced from the point $((x, z)=(0,-18.5))$ far away from the wave active region. At each time, the thermal spread is much larger than in the upstream electrons (dashed curves) in both the parallel and perpendicular directions. This is due to the energization process in the reconnection exhaust. In particular, a flat-top type of distribution is produced in the parallel direction due to the counter-streaming components along the field line, which is in good agreement with the observations typical in the boundary region between the current sheet and magnetospheric lobe (Asano et al., 2008). At $t \omega_{\mathrm{ci}}=106$ (green curves), before the whistler emissions and during the secondary reconnection, the electrons typically have a temperature anisotropy of $T_{\mathrm{e} \perp} / T_{\mathrm{e} \|}<1$ in this region. The high-energy electrons seen at $v_{\|} / V_{\mathrm{A}} \lesssim-20$ are generated in the course of the secondary reconnection. On the other hand, at $t \omega_{\mathrm{ci}}=114$ (red curves) after the secondary reconnection and during the whistler emissions, the electron temperature is increased in the perpendicular direction because of the convection of the perpendicularly heated electrons with $T_{\mathrm{e} \perp} / T_{\mathrm{e} \|}>1$. The whistler waves triggered by the temperature anisotropy scatter the electrons in a pitch angle. As a result, the high-energy electrons with $v_{\|} / V_{\mathrm{A}} \lesssim-20$ are drastically increased, even though the secondary reconnection is over. This high-energy component quickly disappears at $t \omega_{\mathrm{ci}}=120$ (blue curves) after the whistler activities are suppressed.

\section{Conclusions}

The present study proposes a new mechanism for the generation of whistler waves in the course of collisionless magnetic reconnection. The present model can explain the bursty nature of whistler emissions as observed in the Earth's magnetosphere (e.g., Deng and Matsumoto, 2001; Wei et al., 2007; Tang et al., 2013; Graham et al., 2016; Huang et al., 2016; Zhao et al., 2016). The key processes to generate the whistlers are intense perpendicular heating of the electrons in association with plasmoid collision and the subsequent compression of the ambient magnetic field. The perpendicular electron heating occurs during a secondary reconnection forced by the plasmoid collision with the current sheet formed downstream of the main reconnection $x$-line. During the secondary reconnection, the electrons are strongly accelerated in the vicinity of the secondary $x$-line and ejected downstream, leading to perpendicular heating. However, the whistler instability due to the temperature anisotropy is not excited at this moment because of the weak intensity and complicated structure of the magnetic field. Instead, the whistler emissions occur when the further compression of the ambient magnetic field is yielded after the secondary reconnection has ended. Once the whistler waves are excited, the temperature anisotropy is quickly decreased due to the pitchangle scattering, so the wave emissions are suppressed.

The present study has focused on the collision between a plasmoid ejected from the reconnection current layer and the current sheet located at the downstream edge of the reconnection exhaust. However, a similar process for the whistler emissions is also expected in a collision between two plasmoids if the plasmoid size is comparable with or larger than the typical ion scales. During such a collision, the electrons are efficiently accelerated through the secondary reconnection, and a large temperature anisotropy is formed downstream of the electron jets. Subsequent magnetic compression occurs due to the pressure enhancement at the core region of the merged plasmoid, leading to the whistler emissions. In fact, a recent satellite observation shows whistler waves associated with the collision of two plasmoids (Zhao et al., 2016). The whistler waves triggered by plasmoid collision have several characteristics different from the other whistlers in reconnection (Fujimoto and Sydora, 2008; Fujimoto, 2014; Goldman et al., 2014). The plasmoid-induced whistlers are produced in a short-term burst, propagate away from the Equator plane, and have a peak intensity off the Equator plane. In other words, the detection of bursty whistler emissions with these characteristics could be an in- 
dicator of plasmoid collisions and associated particle energization during collisionless magnetic reconnection.

Data availability. The simulation data are available upon request from the author (fujimoto@buaa.edu.cn).

\section{The Supplement related to this article is available online at https://doi.org/10.5194/angeo-35-885-2017- supplement.}

Competing interests. The author declares no conflict of interest.

Acknowledgements. The simulations were carried out with a Fujitsu CX400 at ITC, Nagoya University, with support from the HPC joint research project at Nagoya University.

The topical editor, Minna Palmroth, thanks one anonymous referee for help in evaluating this paper.

\section{References}

Asano, Y., Nakamura, R., Shinohara, I., Fujimoto, M., Takada, T., Baumjohann, W., Owen, C. J., Fazakerley, A. N., Runov, A., Nagai, T., Lucek, E. A., and Rème, H.: Electron flat-top distributions around the magnetic reconnection region, J. Geophys. Res., 113, A01207, https://doi.org/10.1029/2007JA012461, 2008.

Birdsall, C. K. and Langdon, A. B.: Plasma physics via computer simulation, Institute of Physics Publishing, London, 1991.

Burgess, D. and Scholer, M.: Collisionless shocks in space plasmas: structure and accelerated particles, Cambridge University Press, Cambridge, 2015.

Chen, L.-J., Bhattacharjee, A., Puhl-Quinn, P. A., Yang, H., Bessho, N., Imada, S., Mühlbachler, S., Daly, P. W., Lefebvre, B., Khotyaintsev, Y., Vaivads, A., Fazakerley, A., and Georgescu, E.: Observation of energetic electrons within magnetic islands, Nat. Phys., 4, 19-23, https://doi.org/10.1038/nphys777, 2008.

Daughton, W. and Karimabadi, H.: Collisionless magnetic reconnection in large-scale electron-positron plasmas, Phys. Plasmas, 14, 072303, https://doi.org/10.1063/1.2749494, 2007.

Deng, X. H. and Matsumoto, H.: Rapid magnetic reconnection in the Earth's magnetosphere mediated by whistler waves, Nature, 410, 557-560, 2001.

Fujimoto, K.: Time evolution of the electron diffusion region and the reconnection rate in fully kinetic and large system, Phys. Plasmas, 13, 072904, https://doi.org/10.1063/1.2220534, 2006.

Fujimoto, K.: A new electromagnetic particle-in-cell model with adaptive mesh refinement for high-performance parallel computation, J. Comput. Phys., 230, 8508-8526, https://doi.org/10.1016/j.jcp.2011.08.002, 2011.

Fujimoto, K.: Wave activities in separatrix regions of magnetic reconnection, Geophys. Res. Lett., 41, 2721-2728, https://doi.org/10.1002/2014GL059893, 2014.

Fujimoto, K. and Sydora, R. D.: Whistler waves associated in magnetic reconnection, Geophys. Res. Lett., 35, L19112, https://doi.org/10.1029/2008GL035201, 2008.
Fujimoto, M., Shinohara, I., and Kojima, H.: Reconnection and waves: A review with a perspective, Space Sci. Rev., 160, 123 143, https://doi.org/10.1007/s11214-011-9807-7, 2011.

Gary, S. P.: Theory of Space Plasma Microinstabilities, Cambridge Univ. Press, Cambridge, 1993.

Gary, S. P. and Wang, J.: Whistler instability: Electron anisotropy upper bound, J. Geophys. Res., 101, 10749-10754, 1996.

Goldman, M. V., Newman, D. L., Lapenta, G., Andersson, L., Gosling, J. T., Eriksson, S., Markidis, S., Eastwood, J. P., and Ergun, R.: Čerenkov emission of quasiparallel whistlers by fasr electron phase-space holes during magnetic reconnection, Phys. Rev. Lett., 112, 145002, https://doi.org/10.1103/PhysRevLett.112.145002, 2014.

Graham, D. B., Vaivads, A., Khotyaintsev, Y. V., and André, M.: Whistler emission in the separatrix regions of asymmetric magnetic reconnection, J. Geophys. Res., 121, 1934-1954, https://doi.org/10.1002/2015JA021239, 2016.

Hellinger, P., Mangeney, A., and Matthews, A.: Whistler waves in 3D hybrid simulations of quasiperpendicular shocks, Geophys. Res. Lett., 23, 621-624, 1996.

Huang, S. Y., Fu, H. S., Yuan, Z. G., Vaivads, A., Khotyaintsev, Y. V., Retino, A., Zhou, M., Graham, D. B., Fujimoto, K., Sahraoui, F., Deng, X. H., Ni, B., Pang, Y., Fu, S., Wang, D. D., and Zhou, X.: Two types of whistler waves in the hall reconnection region, J. Geophys. Res., 121, 6639-6646, https://doi.org/10.1002/2016JA022650, 2016.

Hull, A. J., Muschietti, L., Oka, M., Larson, D. E., Mozer, F. S., Chaston, C. C., Bonnell, J. W., and Hospodarsky, G. B.: Multiscale whistler waves within Earth's perpendicular bow shock, J. Geophys. Res., 117, A12104, https://doi.org/10.1029/2012JA017870, 2012.

Kennel, C. F. and Petschek, H. E.: Limit on stably trapped particle fluxes, J. Geophys. Res., 71, 1-27, 1966.

Lengyel-Frey, D., Hess, R. A., MacDowall, R. J., Stone, R. G., Lin, N., Balogh, A., and Forsyth, R.: Ulysses observations of whistler waves at interplanetary shocks and in the solar wind, J. Geophys. Res., 101, 27555-27564, 1996.

Olson, J. V., Holzer, R. E., and Smith, E. J.: High-frequency magnetic fluctuations associated wit the Earth's bow shock, J. Geophys. Res., 74, 4601-4617, 1969.

Ossakow, S. L., Ott, E., and Haber, I.: Nonlinear Evolution of Whistler Instabilities, Phys. Fluids, 15, 2341, https://doi.org/10.1063/1.1693875, 1972.

Rodriguez, P. and Gurnett, D. A.: Electrostatic and electromagnetic turbulence associated with the Earth's bow shock, J. Geophys. Res., 80, 19-31, 1975.

Scholer, M., Shinohara, I., and Matsukiyo, S.: Quasi-perpendicular shocks: Length scale of the cross-shock potential, shock reformation, and implication for shock surfing, J. Geophys. Res., 108, 1014, https://doi.org/10.1029/2002JA009515, 2003.

Schreiner, C., Kilian, P., and Spanier F.: Particle scattering off of right-handed dispersive waves, Astrophys. J., 834, 161, https://doi.org/10.3847/1538-4357/834/2/161, 2017.

Tang, X., Cattell, C., Dombeck, J., Dai, L., Wilson III, L. B., Breneman, A., and Hupach, A.: THEMIS observations of the magnetopause electron diffusion region: Large amplitude waves and heated electrons, Geophys. Res. Lett., 40, 2884-2890, https://doi.org/10.1002/grl.50565, 2013. 
Uchino, H., Kurita, S., Harada, Y., Machida, S., and Angelopoulos, $\mathrm{V}$.: Waves in the innermost open boundary layer formed by dayside magnetopause reconnection, J. Geophys. Res., 122, 32913307, https://doi.org/10.1002/2016JA023300, 2017.

Wang, R., Lu, Q., Nakamura, R., Huang, C., Du, A., Guo, F., Teh, W., Wu, M., Lu, S., and Wang, S.: Coalescence of magnetic flux ropes in the ion diffusion region of magnetic reconnection, Nat. Phys., 12, 263-267, https://doi.org/10.1038/NPHYS3578, 2016.

Wei, X. H., Cao, J. B., Zhou, G. C., Santolík, O., Rème, H., Dandouras, I., Cornilleau-Wehrlin, N., Lucek, E., Carr, C. M., and Fazakerley, A.: Cluster observations of waves in the whistler frequency range associated with magnetic reconnection in the Earth's magnetotail, J. Geophys. Res., 112, A10225, https://doi.org/10.1029/2006JA011771, 2007.
Zhang, Y., Matsumoto, H., Kojima, H., and Omura, Y.: Extremely intense whistler mode waves near the bow shock: Geotail observations, J. Geophys. Res., 104, 449-461, 1999.

Zhao, Y., Wang, R., Lu, Q., Du, A., Yao, Z., and Wu, M.: Coalescence of magnetic flux ropes observed in the tailward high-speed flows, J. Geophys. Res., 121, 10898-10909, https://doi.org/10.1002/2016JA023526, 2016. 